\title{
Impact of Earth radiation pressure on GPS position estimates
}

\author{
C. J. Rodriguez-Solano • U. Hugentobler • \\ P. Steigenberger $\cdot$ S. Lutz
}

Received: 31 March 2011 / Accepted: 20 September 2011 / Published online: 13 October 2011

(C) Springer-Verlag 2011

\begin{abstract}
GPS satellite orbits available from the International GNSS Service (IGS) show a consistent radial bias of up to several $\mathrm{cm}$ and a particular pattern in the Satellite Laser Ranging (SLR) residuals, which are suggested to be related to radiation pressure mismodeling. In addition, orbit-related frequencies were identified in geodetic time series such as apparent geocenter motion and station displacements derived from GPS tracking data. A potential solution to these discrepancies is the inclusion of Earth radiation pressure (visible and infrared) modeling in the orbit determination process. This is currently not yet considered by all analysis centers contributing to the IGS final orbits. The acceleration, accounting for Earth radiation and satellite models, is introduced in this paper in the computation of a global GPS network (around 200 IGS sites) adopting the analysis strategies from the Center for Orbit Determination in Europe (CODE). Two solutions covering 9 years (2000-2008) with and without Earth radiation pressure were computed and form the basis for this study. In previous studies, it has been shown that Earth radiation pressure has a non-negligible effect on the GPS orbits, mainly in the radial component. In this paper, the effect on the along-track and cross-track components is studied in more detail. Also in this paper, it is shown that Earth radiation pressure leads to a change in the estimates of GPS ground station positions, which is systematic over large regions of the Earth. This observed "deformation" of the Earth is towards North-South and with large scale patterns that repeat six times per GPS draconitic year (350 days),
\end{abstract}

C. J. Rodriguez-Solano $(\varangle) \cdot$ U. Hugentobler · P. Steigenberger Institut für Astronomische und Physikalische Geodäsie, Technische Universität München, 80333 Munich, Germany e-mail: rodriguez@bv.tum.de

\section{S. Lutz}

Astronomisches Institut, Universität Bern, 3012 Bern, Switzerland reaching a magnitude of up to $1 \mathrm{~mm}$. The impact of Earth radiation pressure on the geocenter and length of day estimates was also investigated, but the effect is found to be less significant as compared to the orbits and position estimates.

Keywords GPS $\cdot$ Albedo $\cdot$ Precise orbit modeling $\cdot$ Spectra of GPS position estimates

\section{Introduction}

The International GNSS Service (IGS, Dow et al. 2009) final orbits have reached an internal precision of around $2.5 \mathrm{~cm}$ for GPS and $5 \mathrm{~cm}$ for GLONASS satellites ${ }^{1}$ from a level about an order of magnitude larger in the mid 1990s. The progress can be attributed to understanding the errors and improving the models affecting the GNSS technique, including those related to precise orbit modeling. Despite the performance attained, however, some problems remain in the orbits but as well as in the position estimates of GPS tracking stations.

The orbits of the two GPS satellites equipped with laser retro reflector arrays (LRA) show a consistent radial bias of up to several $\mathrm{cm}$, when compared with the Satellite Laser Ranging (SLR) measurements, known as the GPS-SLR orbit anomaly. This bias was observed for the Center for Orbit Determination in Europe (CODE) $)^{2}$ final orbits with a magnitude of 3-4 cm by Urschl et al. (2007). More recent

\footnotetext{
$\overline{1}$ http://igscb.jpl.nasa.gov/components/prods.html, accessed on 25 August 2011.

${ }^{2}$ CODE is a consortium formed by: the Astronomical Institute of the University of Bern (AIUB, Bern, Switzerland), the Swiss Federal Office of Topography (swisstopo, Wabern, Switzerland), the Federal Agency for Cartography and Geodesy (BKG, Frankfurt am Main, Germany), and the Institut für Astronomische und Physikalische Geodäsie of the Technische Universität München (IAPG, Munich, Germany).
} 


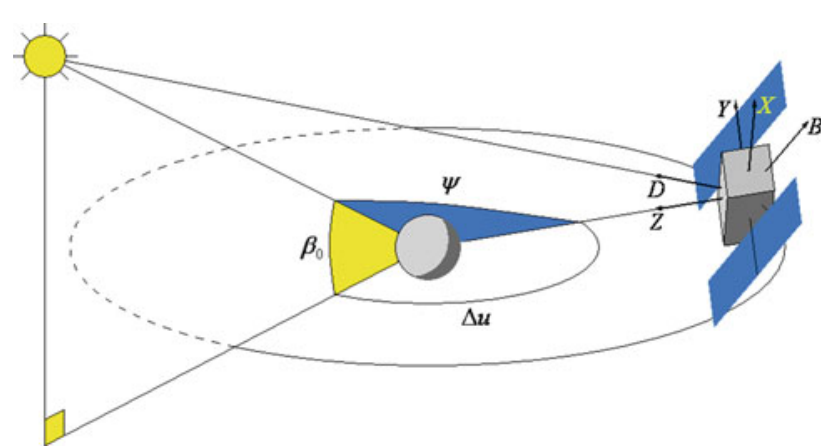

Fig. 1 Relative geometry of Sun, Earth and GPS satellite

comparisons between GPS and SLR measurements (for various IGS analysis centers, Bar-Sever et al. 2009) show a smaller bias of 12-22 $\mathrm{mm}$ with associated scatters of 16$25 \mathrm{~mm}$. These figures can be used as a measure of the current radial accuracy of IGS final orbits. In addition, orbit related frequencies were identified in geodetic time series such as apparent geocenter motion by Hugentobler et al. (2006) and station displacements derived from GPS tracking data by Ray et al. (2008). Particularly, an anomalous frequency of 1.04 cpy (cycles per year) was found, corresponding to a period of about 350 days which is very similar to the GPS draconitic year, the repeat period of the Sun with respect to the satellite constellation.

Urschl et al. (2007) plotted the GPS-SLR residuals as a function of the position of the Sun with respect to the satellite, more specifically in a $\left(\beta_{0}, \Delta u\right)$ reference frame, where $\beta_{0}$ is the elevation angle of the Sun above the orbital plane and $\Delta u$ is the argument of latitude of the satellite with respect to the argument of latitude of the Sun (see Fig. 1). The plot of Urschl et al. (2007) shows a peculiar pattern indicating GPS orbit modeling deficiencies, and the radiation pressure caused by the Earth albedo (not considered at that time in the GPS orbit determination) was mentioned as one of the possible causes to be investigated. Moreover, since the orbit perturbations caused by Earth radiation pressure depend on the position of Sun, Earth and satellite, neglecting Earth radiation pressure is also a good candidate for causing the observed anomalous frequencies in the geodetic time series.

The mathematical formulation used in our study for the computation of Earth radiation pressure (Sect. 2.2) is based on the model of Knocke et al. (1988). The construction of the satellite models for the interaction with Earth radiation (Sect. 2.3) is based on the information contained in Fliegel et al. (1992) and Fliegel and Gallini (1996), who developed solar radiation pressure models for GPS satellites. More recently, Ziebart et al. (2007) and Rodriguez-Solano et al. (2010) studied the impact of Earth radiation pressure on GPS orbits (mainly in the radial component) and on GPS-SLR residuals (see also Sect. 4). In the current paper, we include the acceleration due to Earth radiation pressure (visible and infrared, Sect. 2.4) acting on GPS satellites in the orbit deter- mination process. Two solutions covering nine years (20002008 ) with and without Earth radiation pressure were computed (Sect. 3). This allows to study the impact on the GPS orbits (mainly focussing on the along- and cross-track components, Sect. 4) as well as on GPS derived geodetic parameters like estimated station positions (Sect. 5), geocenter coordinates and length of day (Sect. 6).

\section{Models}

\subsection{Selection of models}

In a previous work (Rodriguez-Solano et al. 2010), Earth radiation and GPS satellite models of increasing complexity were developed and tested. From that study, the key factors of the models have been identified. For the Earth radiation, it was found that the use of an analytical model (based on constant albedo) or one based on numerical integration of Earth's actual reflectivity and emissivity data, give similar results for the irradiance acting on the GPS satellites (difference of up to $10 \%$ ), mainly due to the high altitude of these space vehicles. As data from the Clouds and Earth's Radiant Energy System (CERES, Wielicki et al. 1996) satellite mission are available since the beginning of $2000^{3}$, the numerical approach was chosen (see Sect. 2.2). Concerning the satellite model, use of a box-wing model is a key factor since the variation of the solar panels with respect to the Earth is very important, whereas a simple cannon-ball model is highly inaccurate. The accuracy of the modeled optical properties and the detailed structure of the satellite have a lower impact on the acceleration than the use of a box-wing model, but they are still important enough to be considered (see Sect. 2.3). Further details of the models may be found in Rodriguez-Solano (2009).

\subsection{Earth radiation}

The mathematical formulation of the Earth radiation model used for this study is the same as the one proposed by Knocke et al. (1988) for computing the irradiance received by the satellite due to the Earth's reflected (visible) and emitted (infrared) radiation. It is assumed that the Earth reflects and emits radiation in a purely diffuse way as a Lambertian sphere. The main steps included in the model are: (1) Determine the solar irradiance received by each surface element of the Earth (grid of $2.5^{\circ} \times 2.5^{\circ}$ ). (2) Compute the irradiance received by the satellite based on the reflectivity and emissivity coefficients (from NASA's CERES project, also used by Ziebart et al. 2004 but not yet by Knocke et al. 1988) for

\footnotetext{
3 http://eosweb.larc.nasa.gov/PRODOCS/ceres/level3_es4_table. html, accessed on 29 March 2011.
} 
each Earth's surface element. (3) Compute the Earth radiation pressure caused by the interaction between the irradiance from each surface element of the Earth with the satellite model.

\subsection{GPS satellites}

The physical description of the interaction between radiation and the surfaces of the satellite is provided by Fliegel et al. (1992). It is based on the optical properties of the surface, e.g., specularity and reflectivity or equivalently the fraction of reflected, absorbed and diffused photons which should sum up to one (Milani et al. 1987). The dimensions and optical properties are given for Blocks I and II/IIA GPS satellites in the mentioned paper and for Block IIR satellites in Fliegel and Gallini (1996). These two papers are the basis for constructing our box-wing satellite model. They also provide a priori models (ROCK) for solar radiation pressure for precise geodetic applications. However, nowadays no a priori model or purely empirical models are used due to the lower performance of the ROCK models compared to the empirical models. For this study, the CODE empirical model for solar radiation pressure (see also Sect. 3, Beutler et al. 1994) was used with no a priori model.

To complete the box-wing model, the nominal attitude law of the satellite must be considered, i.e., ensuring that the navigation antennas always point to the geocenter and that the solar panels always point to the Sun. The nominal attitude law is correct in most cases. Only when the satellite is in eclipse season and in the Earth's shadow as at orbit noon , is this no longer true (Bar-Sever 1996; Kouba 2009). However, non-nominal orbit noon and midnight maneuvers are not yet considered in our model. With the assumption of nominal attitude, the Earth radiation and satellite models have a main dependency on the angle $\psi$ formed by satellite, Earth and Sun, as shown in Fig. 1, which can be simply written as $\cos (\psi)=\cos \left(\beta_{0}\right) \cos (\Delta u)$.

Finally, the thrust of the navigation antennas, as reported by Ziebart et al. (2004), was also included in the satellite model. An approximate value of 80 Watts of antenna transmission power was used for all GPS satellites (Block II/IIA and Block IIR). In previous studies (Ziebart et al. 2004; Rodriguez-Solano et al. 2010), the thrust of the navigation antennas was found to cause a non-negligible effect for GPS satellite orbits. It introduces a constant radial acceleration of around $2.7 \times 10^{-10} \mathrm{~m} / \mathrm{s}^{2}$ for Block IIA satellites, a magnitude which is comparable to the minimum radial acceleration due to Earth radiation pressure (around $4 \times 10^{-10} \mathrm{~m} / \mathrm{s}^{2}$ ).

\subsection{Acceleration}

By combining the irradiance from each surface element of the Earth with the satellite model, a force acting on the satellite is
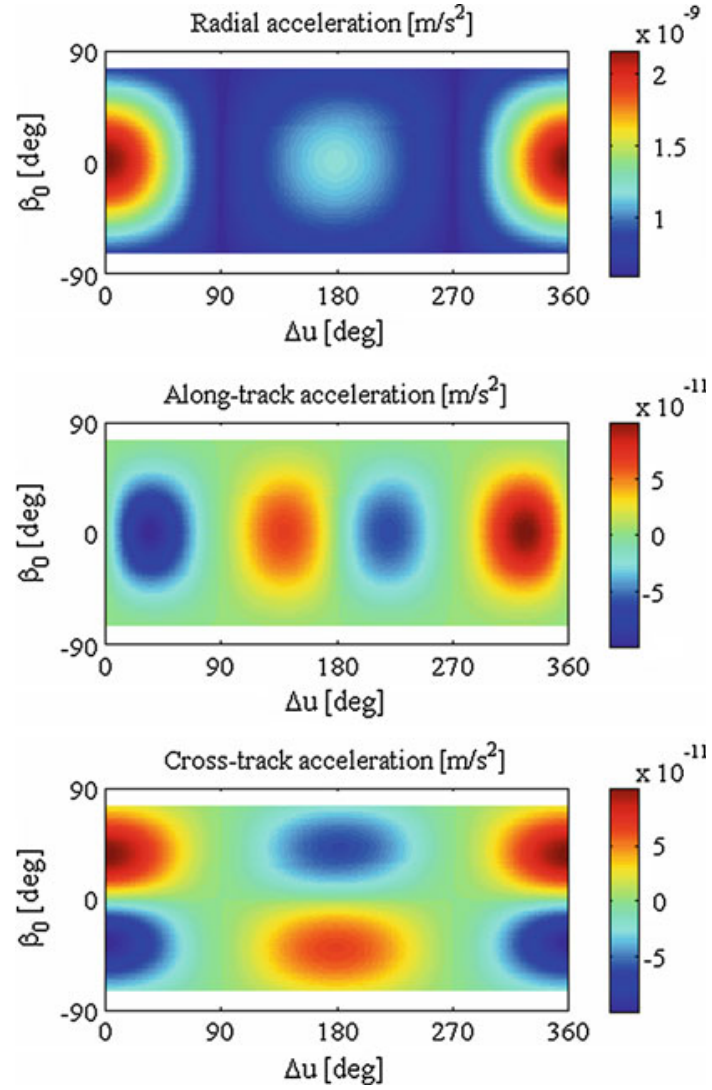

Fig. 2 Acceleration acting on Block IIA satellites due to Earth radiation pressure plus antenna thrust, in a Sun-fixed reference frame. When $\Delta u$ equals $180^{\circ}$, the satellite is above the shadowed side of the Earth

obtained. By integrating these forces over the part of the Earth visible to the satellite and dividing by the mass of the satellite, the acceleration due to Earth radiation pressure for a specific satellite position is obtained. In Fig. 2, the resulting acceleration is shown for a box-wing GPS Block IIA satellite model in a Sun-fixed reference frame $\left(\beta_{0}, \Delta u\right)$ for the radial, alongtrack and cross-track components. To produce this plot, an analytical Earth radiation model was used assuming a globally constant albedo of 0.3 as well as only a radial impact direction of the irradiance reaching the satellite. This was done for computational efficiency. The acceleration used to calculate the GPS satellite orbits was, however, based on the numerical Earth radiation model (with CERES satellite data, Sect. 2.2).

For the radial component of the acceleration (Fig. 2), the maximum at $\Delta u=0^{\circ}$ and $\beta_{0}=0^{\circ}$ corresponds to the point where Sun, satellite and Earth are exactly aligned, with the spacecraft above the Sun-facing side of the Earth. Note that for $90^{\circ}<\Delta u<270^{\circ}$ the satellite is mainly above the night hemisphere of the Earth. At $\Delta u=180^{\circ}$ and $\beta_{0}=0^{\circ}$ it is in total shadow, where one finds a secondary maximum of the radial acceleration since the solar panels are maximally exposed to the infrared radiation of the Earth. This last 
feature would not be present for a cannon-ball model with constant cross-section, since the solar panels change their orientation a lot with respect to the Earth over one revolution. This change of orientation is also responsible for the minima at $\Delta u=90^{\circ}$ and $\Delta u=270^{\circ}$, where the geocentric directions to the satellite and to the Sun are orthogonal and the exposure of the solar panels to Earth radiation is almost zero.

The acceleration observed in the along-track and crosstrack components in Fig. 2 is an effect of the solar panels; i.e., with a cannon-ball model we would not see these particular patterns. Moreover, the along-track acceleration has a period of twice per revolution while the cross-track period is only once per revolution. Also interesting is the change of sign in the cross-track acceleration with the sign of the $\beta_{0}$ angle.

\section{Processing strategy}

The Earth radiation pressure model as described in Sect. 2.2 and 2.3 was implemented in the Bernese GPS Software (Dach et al. 2007). Adapting the strategy of CODE as described by Steigenberger et al. (2006, 2011), 9 years (2000-2008) of GPS ground tracking data were processed, using around 200 IGS stations. Two solutions were computed, one including Earth radiation pressure and one without. No net rotation (NNR) and no net translation (NNT) conditions were applied with respect to the IGS05 (Ferland 2006) reference frame for a subset of up to 125 tracking stations. The last condition is required to estimate the offset between the Earth's center of mass, as sensed by the satellites, and the reference frame origin. The resulting orbits and station coordinates are then obtained in the terrestrial reference frame and the respective comparisons (Sects. 4 and 5) are not affected by the apparent changes of the geocenter. Moreover, the impact of Earth radiation pressure on the apparent geocenter can be studied independently (Sect. 6).

The solar radiation pressure effect (in both solutions) was modeled by estimating five empirical parameters (per satellite and per day) and with no a priori model. The five empirical parameters were proposed by Beutler et al. (1994) and are basically the following: three constants in the $D, Y$ and $B$ directions (see Fig. 1) and two periodic (once per revolution) in the $B$ direction. Additionally, according to the CODE processing strategy, three pseudo-stochastic pulses once per revolution in the radial, along-track and cross-track directions were estimated (individually constrained for each direction). Despite having 9-year solutions, in most of the plots presented in the following sections just the year 2007 is shown to keep the figures simple. The full 9-year solutions are mainly used for computing the spectra of daily position estimates and geocenter.
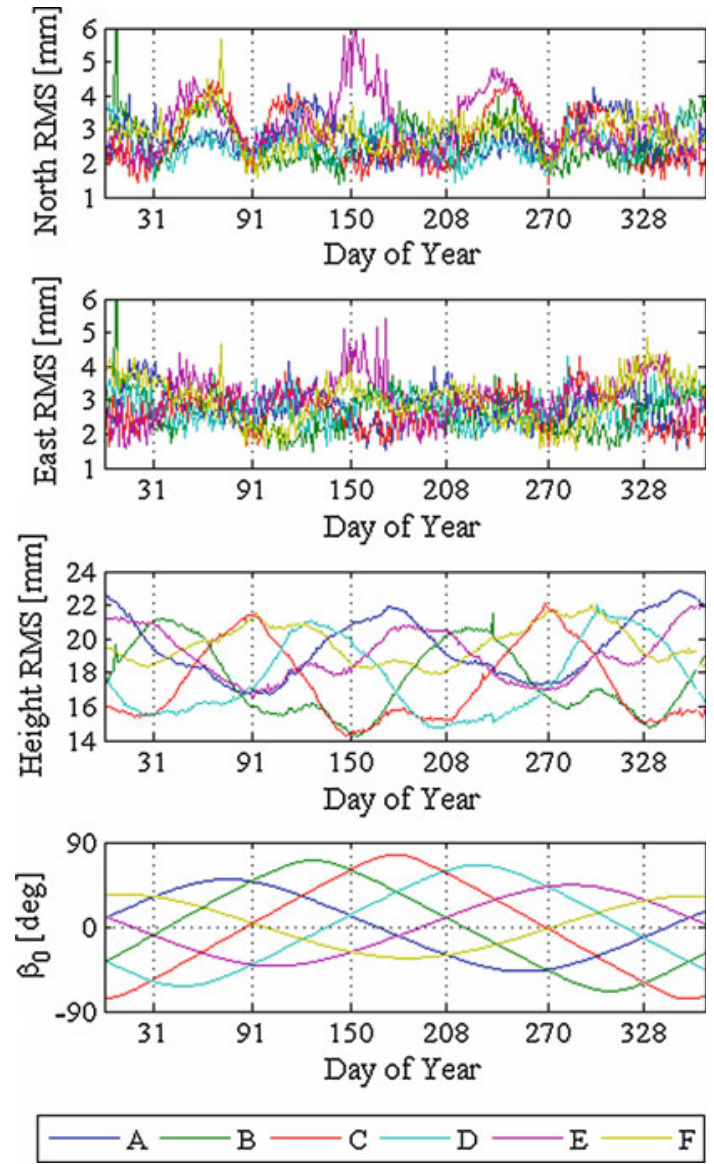

Fig. 3 Top three RMS of GPS orbit differences per orbital plane (in color) and per day of year for 2007. Bottom Sun elevation angle above the GPS orbital planes (in color)

\section{Impact on GPS orbits}

The orbit differences presented in this section were obtained by comparing the orbits of all GPS satellites computed with and without Earth radiation pressure. The results in Figs. 3 and 4 are plotted in a local Earth-fixed reference frame (North, East, height) rather than in an orbit-fixed reference frame (radial, along-track, cross-track) in order to highlight the relation of the orbit perturbations due to Earth radiation pressure and the position estimates of GPS ground tracking stations (see Sect. 5).

The most prominent effect of Earth radiation pressure on the GPS orbits is a radial offset of 1-2 cm, observable in the height component of Figs. 3 and 4. As already mentioned by Ziebart et al. (2007), this effect reduces the GPS-SLR range discrepancy by $1-2 \mathrm{~cm}$, from which around $0.5 \mathrm{~cm}$ can be attributed to the antenna thrust (Sect. 2.4, Rodriguez-Solano et al. 2010). The reason for this radial reduction of orbits is that GPS measurements, being essentially angular measurements due to required clock synchronization, mainly determine the mean motion of the satellite. As a matter of fact, a 

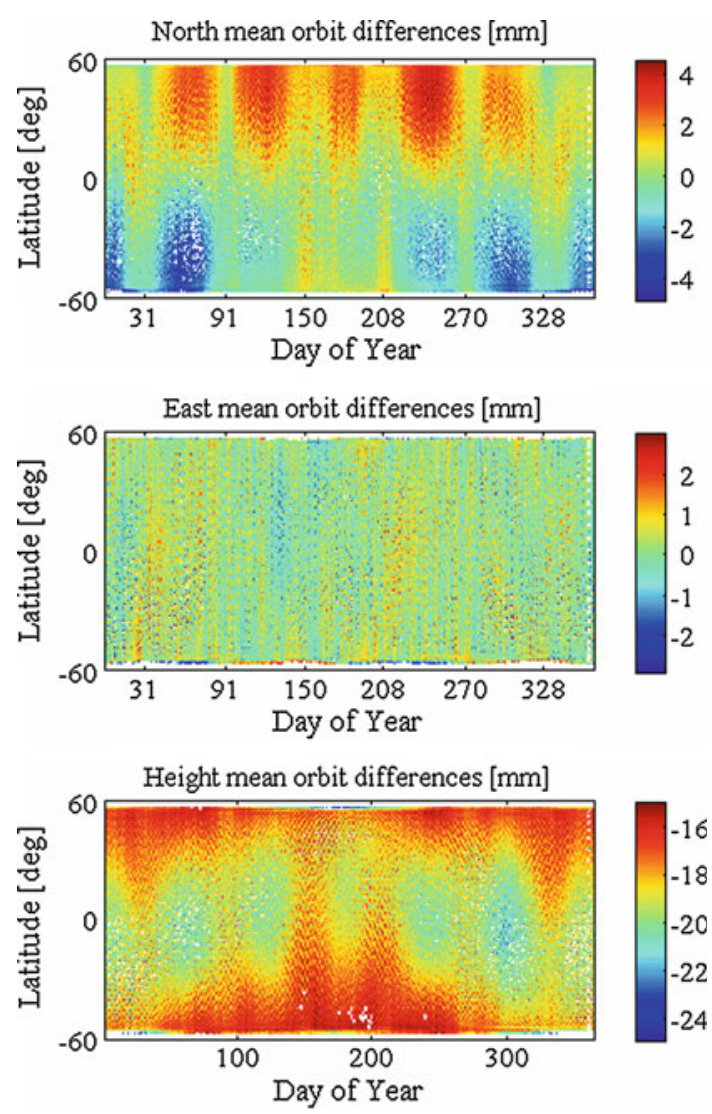

Fig. 4 Mean orbit differences of all GPS satellites plotted as a function of latitude and day of year (2007)

constant positive radial acceleration (equivalent to a reduction of GM, the product of the gravitational constant and the mass of the Earth) decreases the orbital radius according to Kepler's third law (Rodriguez-Solano et al. 2010). From Fig. 3, one can also note the dependency of the height RMS orbit differences with respect to the Sun elevation angle above (or below) the orbital plane $\left(\beta_{0}\right)$. Consequently, this kind of perturbation should have a main repeat period close to half of the GPS draconitic year, that is about 350/2 days. The subdaily dependency of the orbit differences on the $\Delta u$ angle was averaged when Figs. 3 and 4 were produced, since the differences are presented as RMS and mean values per day for the corresponding figures.

However, the North and East RMS orbit differences in Fig. 3 do not seem to have a correlation with the $\beta_{0}$ angle of the respective planes, but rather to the combined effect of the $\beta_{0}$ angles of the six orbital planes. More specifically, one finds a minimum in all the orbital planes, repeating six times during 1 year, for the North component RMS orbit differences (with an exception for day 150 and for the orbital plane E). These minima occur when the $\beta_{0}$ angle of two orbital planes get close to zero degrees (shown with dotted lines in Fig. 3), i.e., when the Sun gets close to the intersection line of the two orbital planes. From the acceleration produced by Earth radiation pressure (Fig. 2), we know that the cross-track acceleration is zero for $\beta_{0}=0^{\circ}$, therefore one could expect a minimum in the North or East RMS orbit differences for the two orbital planes with a Sun elevation angle close to zero. However, the other four orbital planes with $\beta_{0}$ angles different from zero also seem to be minimally affected by Earth radiation pressure (mainly the North component) and have minima at the same periods. The mechanism for this behavior is not yet understood, but it could be further investigated by e.g. excluding the satellites from one or two orbital planes in the solution computation.

By taking the mean of the orbit differences for all GPS satellites for a given latitude (one degree intervals) and day of year, one gets a very interesting result (Fig. 4). For example, the orbit height differences follow the position of the Sun with respect to the Earth over the year, something that is expected since the Earth radiation at the satellite reaches its highest values if there is an alignment of Sun, satellite and Earth. For the East component we find almost no signal (compared to height and North) in the orbit differences. In the North orbit differences we obtain a very similar pattern as the one of Fig. 3, but in addition we observe that the differences are positive for the northern hemisphere and negative for the southern one. This can be interpreted as a change in the inclination of the orbits due to Earth radiation pressure. In fact, the inclination changes by around $1 \times 10^{-8}$ degrees, which corresponds to $4.6 \mathrm{~mm}$ in the cross-track orbit positions (Fig. 4).

\section{Impact on GPS station position estimates}

Earth radiation pressure mismodeling causes a very particular perturbation at the millimeter level in the daily position estimates of GPS ground tracking stations. Figure 5 shows horizontal displacement vectors between solutions with and without modeled Earth radiation pressure. This is shown for 2 days of 2007, one where the North orbit differences (see Fig. 3) are large (day 61) and one where they are minimal (day 91). For day 61, a "deformation" of the Earth in north- and southwards direction is visible. Moreover, the two characteristic patterns in Fig. 5 alternate six times per GPS draconitic year (350 days). There are also differences in the station height components (not shown), but their magnitude is smaller than for the North component, which are the most prominent. The comparison of the station positions based on solutions with and without Earth radiation pressure, shown in Fig. 5, was performed using a 7-parameter Helmert transformation. The scale difference is around $0.14 \mathrm{ppb}$ (parts per billion), or a uniform height shift of about $0.9 \mathrm{~mm}$. In Fig. 6, the translation parameters are shown. The shift in the $Z$-axis is related to the distribution of GPS stations: as more 

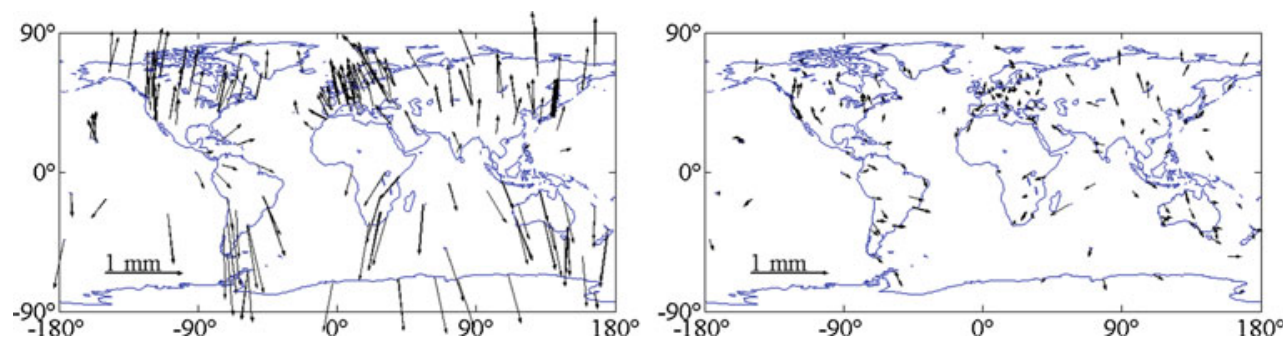

Fig. 5 Change in the estimated horizontal GPS ground station positions (about 200 IGS sites) due to Earth radiation pressure for day 61 (left) and day 91 (right) of year 2007

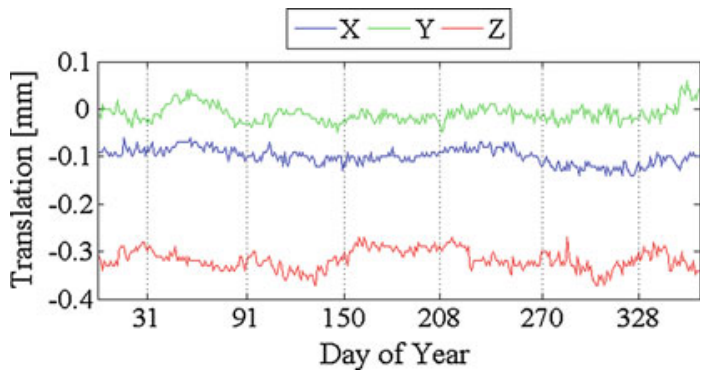

Fig. 6 Helmert translation parameters between GPS position solutions with and without Earth radiation pressure for year 2007

stations are located in the northern hemisphere these dominate the no net translation condition imposed in the solution (see also Sect. 3). Without the Helmert transformation we would observe in Fig. 5, the northern stations were almost unchanged and the southern ones were largely displaced. The translation in the $X$-axis is accompanied by a rotation around the same axis of about $-2 \mu$ as, the rotations around $\mathrm{Y}$ and $\mathrm{Z}$ are both within $\pm 1 \mu$ as.

To demonstrate the correlation between the effects observed in Sect. 4 and the ground station displacements, we plot the mean of the North component differences (of both orbits and stations) as a function of day of year and latitude (one degree intervals). Figure 7 shows basically the same information as Fig. 4 but plotted in a different way: the differences are shown along the ordinate axis while colors indicate the latitude. This representation is more appropriate to display station displacements as a plot similar to Fig. 4 would leave large gaps in the southern hemisphere. The results show the strong correlation between the orbit perturbations and the station displacements. However, the magnitude of the orbit differences is around five times larger than the station differences. If we imagine a GPS satellite placed almost directly over a ground station, both in a middle northern latitude, the satellite would be "pushed" by Earth radiation pressure northwards as well as the station. This relation is almost direct; e.g., if we compute the ratio between the satellite's semimajor axis and the Earth radius $(26,560 \mathrm{~km} / 6,371 \mathrm{~km})$ we obtain a ratio of 4.17 , very close to what we observe in Fig. 7. The situation is of course much more complicated. Nevertheless,
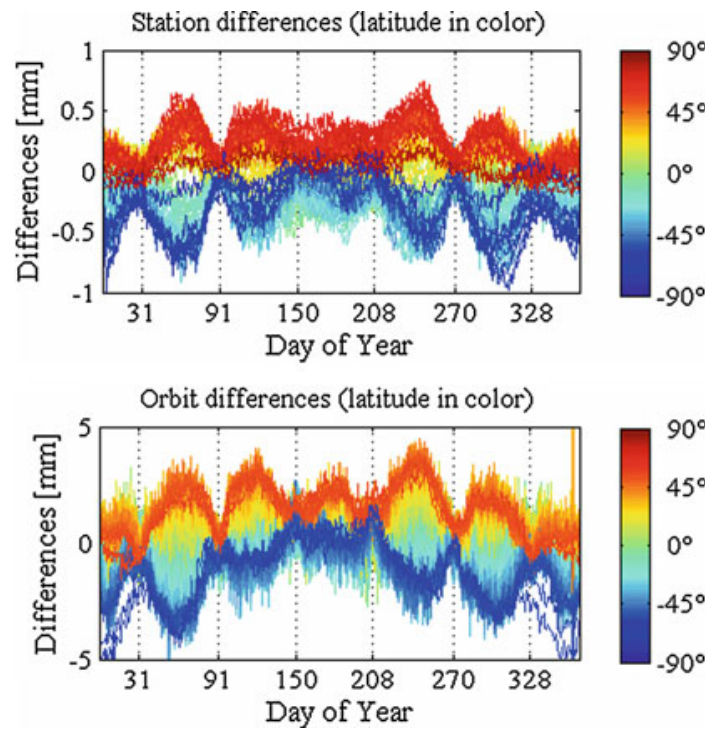

Fig. 7 North component of the mean stations differences (top) and orbit differences (bottom) for year 2007, as a function of day of year and color-coded latitude

we are then in a situation where a small change in the alongtrack or cross-track position of the GPS satellites (which can not be absorbed by the clocks as in the case of the radial component) leads to an almost direct effect on the position estimates of GPS ground tracking stations.

Furthermore, the observed "deformation" of the Earth changes the power spectrum of the North component of GPS daily position estimates (shown in Fig. 8). This figure is based on nine years of tracking data from around 200 globally distributed IGS stations. By introducing Earth radiation pressure we obtain a reduction mainly in the sixth peak of the power spectrum of the North component, located at approximately $6 \times 1.04$ cpy. This frequency is also noticeable in Figs. 3, 4 and 7 (and in Fig. 5 if it would be animated), where we can see a systematic pattern of six cycles over one GPS draconitic year (around 350 days), the repeat period of the Sun with respect to the satellite constellation. As observed in Fig. 8 and also mentioned by Ray et al. (2008), the sixth peak in the North component is one of the sharpest and highest. This peak is reduced from 


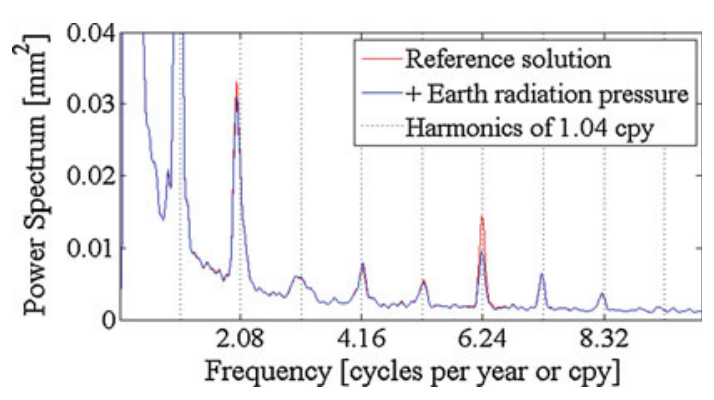

Fig. 8 Power spectrum of the North component of GPS daily position estimates (around 200 IGS sites) from 2000 to 2008, with (blue) and without $(r e d)$ Earth radiation pressure

$0.01443 \mathrm{~mm}^{2}$ to $0.00956 \mathrm{~mm}^{2}$ and after subtracting a noise floor of $0.0016 \mathrm{~mm}^{2}$, we obtain a variance reduction of $38 \%$. This result is very important since it indicates that the solution that includes Earth radiation pressure reduces systematically the anomalous frequency. It also demonstrates that the observed anomalous frequencies in the GPS position time series are related (at least partially) to orbit mismodeling issues. However, the peaks in the East and height power spectrum (not shown) do not exhibit a significant reduction.

The power spectra in this paper were not computed using the Lomb-Scargle Periodogram (Scargle 1982; Press et al. 1992), as done by other authors (Ray et al. 2008; Tregoning and Watson 2009) $)^{4}$. Instead, the power spectrum of the Fast Fourier Transform (FFT, Press et al. 1992) was used and where data is missing, zero padding was employed, since GPS-derived daily positions (station coordinates and geocenter) are evenly spaced. The units of the power spectrum are clear $\left(\mathrm{mm}^{2}\right)$, while the Lomb-Scargle Periodogram (due to normalization) has no units, and finally, the computation time is largely reduced when employing the FFT. Additionally, to compute the mean power spectrum of the 200 IGS stations (Fig. 8) a weighting according to the inverse of the variance was introduced to ensure that the noisier position time series have a lower contribution.

\section{Impact on geocenter and LOD}

The impact of Earth radiation pressure on other geodetic parameters, specifically the geocenter position and the length of day (LOD), was also investigated. For the geocenter we find an impact at the one millimeter level (see Fig. 9), but no clear pattern is visible as for the GPS position esti-

\footnotetext{
4 There are also other methods for computing the power spectrum, e.g., a robust estimation method was used by Collilieux et al. (2007) for identifying the draconitic harmonics in the individual time series of GPS stations.
}

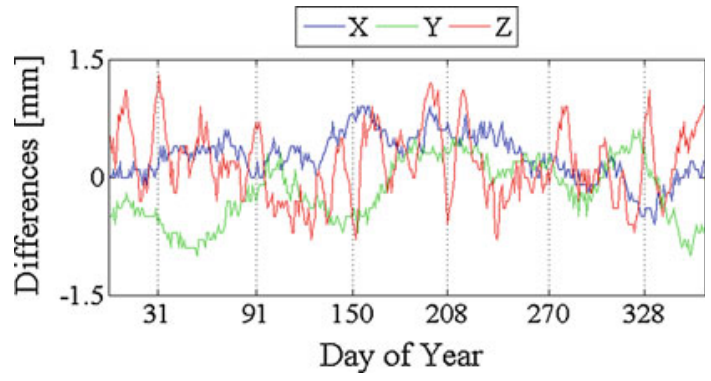

Fig. 9 Geocenter differences between solutions with and without Earth radiation pressure for year 2007

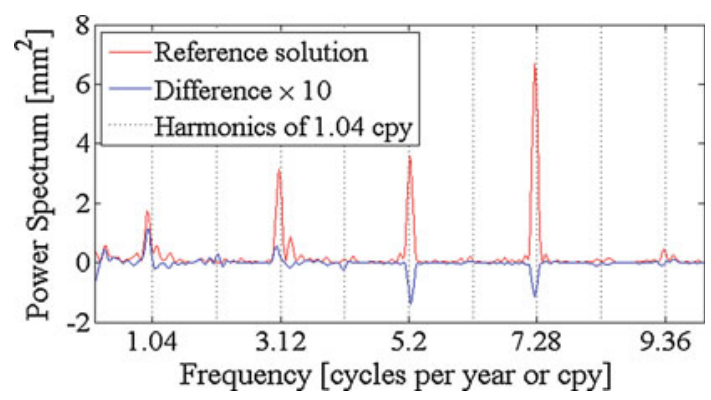

Fig. 10 Power spectrum of the $Z$-component of the GPS-derived geocenter from 2000 to 2008 . The power spectrum difference between the reference solution minus the one including Earth radiation pressure multiplied by ten is shown in blue

mates (Sect. 5). The geocenter is appropriate for investigation since in the $Z$-component a period of 350 days, the GPS draconitic year, corresponding to $1.04 \mathrm{cpy}$, was identified by Hugentobler et al. (2006). The power spectrum of the geocenter time series (without modeling Earth radiation pressure) is shown in Fig. 10. Note that the main anomalous peaks are found at odd multiples $(1,3$, $5,7)$ of 1.04 cpy. By including the Earth radiation pressure a small reduction in the 5th and 7th peaks has been achieved, 3.8 and $1.7 \%$, respectively. Figure 10 shows the difference of the power spectra of the solutions with and without Earth radiation pressure, however multiplied by a factor of 10. A negative sign of the difference, as found for the 5th and 7th peaks, means a reduction of the original power spectrum, while a positive sign means an increase as observed for the 1st and 3rd peaks (by 6.4 and $1.7 \%$, respectively).

Figure 11, finally, shows the difference in the LOD estimates obtained with and without Earth radiation pressure. The impact is of the order of $10 \mu$ s with a pattern that has a period close to 350 days, again, the GPS draconitic year. However the LOD power spectrum derived from GPS measurements does not show significant peaks at harmonics of 1.04 cpy. Also the difference of the power spectra of the LOD estimates only shows small variations at harmonics of 1.04 cpy. 


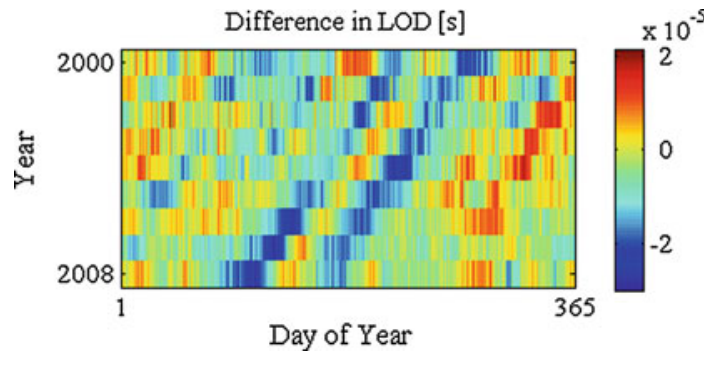

Fig. 11 Difference in length of day (LOD) between the solutions with and without Earth radiation pressure from 2000 to 2008

\section{Conclusions}

The acceleration acting on GPS (i.e. box-wing-like) satellites caused by Earth radiation (visible and infrared) depends mainly on the relative position of each satellite, Earth and Sun. This implies a perturbation in the GPS satellite orbits at harmonics of the GPS draconitic year. The main non-negligible effect on the orbits is a reduction of the height by about 1-2 cm, consistent with the findings of Ziebart et al. (2007). In the other components of the orbits (along-track and cross-track), the effect is about one order of magnitude smaller. When the orbit differences (with and without Earth radiation pressure) are separated by orbital plane, it becomes evident that when the Earth radiation pressure is zero in the cross-track component for two orbital planes, the other four orbital planes are also unaffected, the mechanism for which requires further investigation.

Moreover, in the North component of the orbit differences a particular pattern is found, displacing the orbits (few millimeters) towards North at northern latitudes and South at southern latitudes, with a period of one sixth of the GPS draconitic year. The same pattern is found in the daily position estimates of GPS ground stations at the sub-millimeter level, indicating that a small change in the along-track or cross-track position of the GPS satellites (which can not be absorbed by the clocks like the radial shift) leads to an almost direct effect on the position estimates of GPS ground stations. This "deformation" of the Earth also leads to a reduction in the anomalous spectra of GPS daily position estimates, mainly in the sixth peak of the North component at $6 \times 1.04$ cpy.

The impact of Earth radiation pressure on the geocenter (at the one millimeter level) is less significant. It reduces just slightly some of the anomalous peaks of the geocenter power spectrum and even increases others. For LOD, the impact is of order $10 \mu \mathrm{s}$. The fact that the impact on geocenter and LOD is low, indicates that other more important problems remain in the orbit modeling, in particular for the geocenter. One of the most likely candidates is the solar radiation pressure, which also has a strong dependency on the GPS draconitic year. It is larger in magnitude than the Earth radiation pressure and currently it is considered mainly by applying empirical parameterizations.

Finally, the non-negligible effects of Earth radiation pressure that have been found in the GPS orbits and station position estimates underlines the importance of further improvements in the orbit-modeling techniques. Of particular concern is a better understanding and modeling of nonconservatives forces affecting the satellites, which involve the modeling of the radiation source, the satellite structure (including its attitude) and surface properties. This also justifies the efforts that are invested in this task by different groups of scientists.

The subroutines to compute the acceleration due to Earth radiation pressure are available at: http://www.iapg.bv.tum. de/albedo/. The subroutines contain the Earth radiation models (analytical and numerical with CERES data) as well as the box-wing models for GNSS satellites (GPS and GLONASS).

Acknowledgments The authors gratefully acknowledge the International GNSS Service (IGS, Dow et al. 2009) for providing the high quality data needed for this study. The comments and suggestions by Jim Ray (NOAA) and two other reviewers are greatly appreciated. We acknowledge the support of the TUM Graduate School.

\section{References}

Bar-Sever Y, Davis JL, Dach R, Flohrer C, Herring T, Ray J, Slater JA, Thaller D (2009) Impact of SLR tracking on GPS. In: Pavlis EC (ed) International Technical Laser Workshop on SLR Tracking of GNSS Constellations, http://acc.igs.org/orbits/ slr_track_gps_ilrs09-PP.pdf, Position Paper

Bar-Sever YE (1996) A new model for GPS yaw attitude. J Geod 70(11):714-723. doi:10.1007/BF00867149

Beutler G, Brockmann E, Gurtner W, Hugentobler U, Mervart L, Rothacher M, Verdun A (1994) Extended orbit modeling techniques at the CODE processing center of the international GPS service for geodynamics (IGS): theory and initial results. Manuscr Geod 19(6):367-386

Collilieux X, Altamini Z, Coulot D, Ray J, Sillard P (2007) Comparison of very long baseline interferometry, GPS, and satellite laser ranging height residuals from ITRF2005 using spectral and correlation methods. J Geophys Res 112(B12403). doi:10.1029/ 2007JB004933

Dach R, Hugentobler U, Fridez P, Meindl M (2007) Bernese GPS Software Version, 5.0. Astronomical Institute, University of Bern

Dow J, Neilan R, Rizos C (2009) The International GNSS Service in a changing landscape of Global Navigation Satellite Systems. J Geod 83(3-4):191-198. doi:10.1007/s00190-008-0300-3

Ferland R (2006) [IGSMAIL-5455]: IGS05 fine tuning. http://igscb.jpl. nasa.gov/mail/igsmail/2006/msg00178.html

Fliegel H, Gallini T (1996) Solar force modeling of block IIR global positioning system satellites. J Spacecr Rockets 33(6):863-866. doi: $10.2514 / 3.26851$

Fliegel H, Gallini T, Swift E (1992) Global positioning system radiation force model for geodetic applications. J Geophys Res 97(B1):559_ 568. doi:10.1029/91JB02564

Hugentobler U, van der Marel H, Springer T (2006) Identification and mitigation of GNSS errors. In: Springer T, Gendt G, Dow JM (eds) 
The International GNSS Service (IGS): Perspectives and Visions for 2010 and beyond, IGS Workshop 2006

Knocke PC, Ries JC, Tapley BD (1988) Earth radiation pressure effects on satellites. In: Proceedings of AIAA/AAS Astrodynamics Conference, pp 577-587

Kouba J (2009) A simplified yaw-attitude model for eclipsing GPS satellites. GPS Solut 13(1):1-12. doi:10.1007/s10291-008-0092-1

Milani A, Nobili AM, Farinella P (1987) Non-Gravitational Perturbartions and Satellite Geodesy. Adam Hilger, Bristol

Press W, Teukolsky S, Vetterling W, Flannery B (1992) Numerical Recipes in Fortran 77: The Art of Scientific Computing, 2nd edn. Cambridge University Press, Cambridge

Ray J, Altamini Z, Collilieux X, Dam T (2008) Anomalous harmonics in the spectra of GPS position estimates. GPS Solut 12(1):55-64. doi:10.1007/s10291-007-0067-7

Rodriguez-Solano CJ (2009) Impact of albedo modelling on GPS orbits. Master's thesis, Technische Universität München. http:// mediatum2.ub.tum.de/doc/1083571/1083571.pdf

Rodriguez-Solano CJ, Hugentobler U, Steigenberger P (2010) Impact of Albedo Radiation on GPS Satellites. In: Geodesy for Planet Earth, International Association of Geodesy Symposia. Springer, Berlin (in press)

Scargle J (1982) Studies in astronomical time series analysis. II. statistical aspects of spectral analysis of unevenly spaced data. Astrophys J 263:835-853. doi:10.1086/160554
Steigenberger P, Rothacher M, Dietrich R, Fritsche M, Rülke A, Vey S (2006) Reprocessing of a global GPS network. J Geophys Res 111(B05402). doi:10.1029/2005JB003747

Steigenberger P, Hugentobler U, Lutz S, Dach R (2011) CODE Contribution to the First IGS Reprocessing Campaign. Tech. Rep. 1/2011, IAPG/TUM. http://mediatum2.ub.tum.de/doc/1078108/1078108. pdf

Tregoning P, Watson C (2009) Atmospheric effects and spurious signals in GPS analysis. J Geophys Res 114, B09403. doi:10.1029/ 2009JB006344

Urschl C, Beutler G, Gurtner W, Hugentobler U, Schaer S (2007) Contribution of SLR tracking data to GNSS orbit determination. Adv Space Res 39:1515-1523. doi:10.1016/j.asr.2007.01.038

Wielicki BA, Barkstrom BR, Harrison EF, Lee RBIII, Smith GL, Cooper JE (1996) Clouds and the Earth's Radiant Energy System (CERES): an Earth observing system experiment. Bull Am Meteorol Soc 77(5):853-868

Ziebart M, Edwards S, Adhya S, Sibthorpe A, Arrowsmith P, Cross P (2004) High precision GPS IIR orbit prediction using analytical non-conservative force models. In: Proceedings of ION-GNSS2004, pp 1764-1770

Ziebart M, Sibthorpe A, Cross P, Bar-Sever Y, Haines B (2007) Cracking the GPS-SLR Orbit Anomaly. In: Proceedings of ION-GNSS2007, pp 2033-2038 